Meta

Journal des traducteurs

Translators' Journal

\title{
Index of Words and Topics in Volume 22
}

Volume 22, numéro 4, décembre 1977

URI : https://id.erudit.org/iderudit/003192ar

DOI : https://doi.org/10.7202/003192ar

Aller au sommaire du numéro

\section{Éditeur(s)}

Les Presses de l'Université de Montréal

ISSN

0026-0452 (imprimé)

1492-1421 (numérique)

Découvrir la revue

Citer ce document

(1977). Index of Words and Topics in Volume 22. Meta, 22(4), 308-309.

https://doi.org/10.7202/003192ar d'utilisation que vous pouvez consulter en ligne.

https://apropos.erudit.org/fr/usagers/politique-dutilisation/ 


\section{INDEX OF WORDS AND TOPICS IN VOLUME 22}

Admission examinations, 2, p. 154.

Air-cylinder hoist, 2, p. 114.

Agenda, 2, p. 277.

Amatol, 4, p, 258.

Amplification, 4, 248.

Area studies, 2, p. 120.

Artistic director, 2, p. 126.

Assistant stage manager, 2, p. 126

Audio-lingual method, 2, p. 119.

Audit, 3, p. 198.

Audit around the computer, 4, p. 282.

Auditor, 3, p. 198.

Beery, 2, p. 134.

Be tuned (to), 3, p. 219.

Bible (translations of the), 2, p. 108.

Binominal phrase, 3, p. 171 .

Biological journals, 4, p. 291

Biology, 3, p. $220 ; 4$, p. 290.

Black powder, 4, p. 259.

Blotto, 2, p. 135

Blued, 2, p. 135.

Body-guard, 3, p. 172.

Bridgehead, 3, p. 172.

Brown shoe admiral, 4, p. 261.

Bunching tables, 2, p. 138.

Bureaucratic translation, 1, p. 38

Business talk, 3, p. 183.

Canada Council, 1, p. 37.

Canada Council Program, 1, p. 43.

Canned, 2, p. 135.

Capacitor, 3, p. 219.

CB, 4, p. 270.

Centre rail, 3 , p. 172.

Chambermaid, 3 , p. 172

Characterization, 3, p. 174, 181.

Citizen's Band, 4, p. 270.

Climax of fuddlement, 2, p. 134.

Cocktail table, 2, p. 137.

Coffee table, 2, p. 137.

Cognitive-referential function, 3, p. 103.

Communication, 2, p. 166

Concentration, 4, p. 248

Consecutive interpreting, 2, p. 122

Construction industry, 4, p. 290.

Container, 4, p. 259.

Count noun, 3 , p. 177

Courtship coloration, 3 , p. 221 .

Covertly errors, 2, p. 105 .

Covert translation, 2, p. 106.

Criteria for quality, 4, p. 243.

Cross-fertilization, 1, p. 69.

Cultural filter, 2, p. 107.

Curricular planning, 2, p. 117.

Cut-away, 4, p. 259.

Dead-drunk, 2, p. 133.

Dead to the world, 2, p. 135.

Degrees of formality, 2, p. 104.

Determiner, 3, p. 172.

Dilution, 4, p. 248.
Director, 2, p. 126.

Dominion, 1, p. 31

Dressing gown, 3, p. 172.

Drunk, 2, p. 132.

Elevated, 2, p. 134.

End table, 2, p. 137, 140.

Energy, 3, p. 225.

English-Canadian verse, 1, p: 42.

English translations of poetry, 1, p. 42.

Equivalency, 4, p. 243.

Equivalent, 2, p. 103.

Essay, 1, p. 42.

Exclamation mark, 3, p. 183.

Expert language translation, 2, p. 119.

Explorers' journals, 1, p. 38.

Feed-back, 2, p. 145.

Fiddler (drung as a), 2, p. 136.

Flying high, 2, p. 134.

Free translation, 4, p. 248.

French definite article, 3, p. 178.

Full, 2, p. 135.

Functions of language, 2, p. 103.

Gain, 4, p. 248.

Genetive form, 3, p. 173, 178.

Geographical terms, 4, p. 285.

Half-seas-over, 2, p. 135.

Happy, 2, p. 134.

Have I (to) a full cargo aboard, 2, p. 135.

Have (to) three sheets in the winds, 2, p. 135.

High, 2, p. 134

Home attitude, 3, p. 183.

Horse power, 3, p. 172.

Hot money, 2, p. 145.

Housemaid, 3, p. 172.

Incentives, 2, p. 145.

Inebriated, 2, p. 133.

Interpreters, 1, p. 122.

Intoxicated, 2, p. 133.

Intralinguistic reading process, 4, p. 245.

Inventory trouble, $3, \mathrm{p} .183$.

ISO, 2, p. 130 .

Jolly, 2, p. 134.

Knocked up, 2, p. 135.

Labour market, 2, p. 118.

Lady's maid, 3, p. 172.

Lamp table, 2, p. 137.

Language and linguistics, 4, p. 290.

Language, interpretation and communication, 2 , p. 166.

Language laboratory, 4, p. 264.

Language training, 2, p. 119.

Language use, 2, p. 104.

Language user, 2, p. 104.

Life sciences, 3, p. 223.

Liquor pond street, 2, p. 135.

Lit, 2, p. 134. 
Literal translation, 1, p. 37 ; 3, p. $235 ; 4$, p. 243.

Literary Translators' Association, 1, p. 43.

Ljudskanove, A.-K., 2, p. 161.

Lord (drunk as a), 2, p. 136.

Loss, 4, p. 248.

Lubricated, 2, p. 135.

Man-of-war, 3, p. 172.

Marketing, 3, p. 212.

Marketing-mix, 3, p. 211.

Mass noun, 3, p. 177.

Matching, 2, p. 145.

Measurement of translation skills, 2, p. 154.

Merchandising, 3, p. 213.

Merry, 2, p. 134.

Mild intoxication, 2, p. 134

Military terms, 4, p. 290.

Misadjustment, 2, p. 145.

Modality, 4, p. 250.

Modifier, 3, p. 179.

Modulation, 4, p. 248

Monitoring, of interpretation, 4, p. 268.

Nailing machine, 4, p. 260.

Nailing machine operator, 4 , p. 260.

Native-language course, 2, p. 120.

Nests of tables, 2, p. 140.

Normalization, 3, p. 174, 180.

Non-cognitive function, $2, \mathrm{p} .103$.

Non-linguistic complementary subject, 2, p. 121.

Oiled, 2, p. 135.

Options and constraints, 4, p. 245.

Oral text reproduction, 2, p. 122.

Orange peel, 3, p. 172 .

Order of the day, 4, p. 276

Otto syenite, 4 , p. 261.

Overtly erroneous errors, 2, p. 105.

Overtranslation, 4, p. 249.

Overt translation, 2, p. 106.

Padding, 3, p. 218.

Paralysed, 2, p. 135.

Permeance, 3, p. 220.

Person-to-person transaction, 3, p. 183.

Pickled, 2, p. 134, 135.

Pinko, 2, p. 135.

Piper (drunk as a), 2, p. 136.

Pleasure principle, $3, \mathrm{p} .171$.

Pointee-talkee, 4, p. 259.

Potato, 3, p. 172 .

Pragmatic meaning, 2, p. 103.

Present fairly, 3, p. 195, 203.

Preservation of meaning, 2, p. 103.

Primed, 2, p. 134.

Producer, 2, p. 126

Psychiatry, 4, p. 285.

Public accountancy, 3, p. 197.

Quality, 2, p. 103.

Quantity (expression of), 3, p. 173.

Quebec fiction, 1, p. 41.

Quebec theatre, 1, p. 42 .

Reduction, 4, p. 248.

Regional studies, 2, p. 120.

Resistivity, 3, p. 218 .

Resistor, 3, p. 219.

Rosy, 2, p. 135.
Schizolinguistics, 4, p. 285.

Science of translation, 1, p. $90 ; 2$, p. 120, 122.

Scientific papers, 4, p. 291.

Selling arguments, 2, p. 145.

Semantic meaning, 2, p. 103

Semiotic model, 4, p. 243.

Simulation of conference situations, 4, p. 268.

Simulation of interpretation, 4, p. 268.

Simultaneout interpreting, 2, p. 123.

Simultaneous interpretation, 4, p. 264.

Simultaneous training, 4, p. 268.

Situational dimensions, 2, p. 104.

Skunk (drunk as a), 2, p. 136 .

Slow operating relay, 3, p. 218.

Social attitude, 2, p. 104 .

Social role relationship, 2, p. 104

Socio-cultural norm, 2, p. 105.

Soused, 2, p. 134.

Specification, 3, p. 174.

Specific conductivity, 3, p. 218.

Speech analysis, 2, p. 122

Speech training, 2, p. 122.

Stage director, 2, p. 126.

Stage manager, 2, p. 126

Standard language, 2, p. 106.

Stare (to), 2, p. 148.

State of beastliness, 2, p. 134.

Substitution procedure, 4, p. 248.

Swivel, 3, p. 172.

Tea table, 2, p. 139.

Textually oriented theory, 4, p. 244.

Textual meaning, 2, p. 103

Textual profile, 2, p. 105 .

Telephone call, 3, p. 183

Theatre, 2, p. 125.

Thread (to), 3, p. 189.

Tight, 2 , p. 134.

Tipsy, 2, p. 133.

Topsy-boozy, 2, p. 135.

Trading partners, 2 , p. 145.

Translating poetry, 2 , p. 147

Translation exercises, 2, p. 119, 121.

Translation price, 1, p. 43 .

Translation quality, 2, p. 103.

Translation theory, 1, p. $89 ; 3$, p. 172

Translatology, 3, p. 172.

Transposition, 4, p. 248.

Treacle, 4, p. 261.

True and correct view, 3, p. 201.

True and fair view, 3, p. 198.

True savings account, 2, p. 145.

Ungrammatical utterances, 3, p. 173.

Unreliable technology, 4, p. 268.

Under the influence, 2, p. 134.

Undertranslation, 4, 249.

Undeterminedness, 4, p. 244.

Venice treacle, 4, p. 261.

Visualized instruction, 4, p. 290.

Waiter, 3, p. 173.

Walkie-talkie, 4, p. 270, 271

Warship, 3, p. 172

Wet, 2, p. 135.

Winey, 2, p. 134.

Woozy, 2, p. 135

Work ethic, 3, p. 171. 